\title{
«ÉRASE UNA VEZ....: LA CREACIÓN DE HISTORIAS COMO HERRAMIENTA DE APRENDIZAJE ACTIVO EN EL GRADO EN RELACIONES LABORALES Y RECURSOS HUMANOS*
}

\author{
«Once Upon a Time...»: Storytelling as an Active-Learning Tool \\ in the Labour Relations and Human Resources' Degree
}

\author{
Elena Fernández del Río \\ Universidad de Zaragoza \\ Orcid: 0000-0002-9645-8109 \\ Alfredo Berges Saldaña \\ Universidad de Zaragoza \\ Orcid: 0000-0001-6055-1929 \\ Pedro José Ramos Villagrasa \\ Universidad de Zaragoza \\ Orcid: 0000-0001-8640-4097
}

\section{ABSTRACT}

La creación de historias o storytelling constituye una herramienta útil en la enseñanza universitaria en cuanto promueve en los estudiantes un aprendizaje más activo. Esto es especialmente relevante en el ámbito de las Relaciones Laborales y

* Este trabajo ha sido llevado a cabo dentro del proyecto de innovación docente (PIIDUZ_16_148) en la Convocatoria de Innovación Docente 2016-2017 de la Universidad de Zaragoza. 
Recursos Humanos (RLRH), ya que la historia creada refleja situaciones concretas relacionadas con el comportamiento humano dentro del ámbito laboral. El objetivo de este trabajo es analizar los resultados obtenidos tras la introducción del storytelling con 111 alumnos matriculados en una asignatura del Grado en RLRH de la Universidad de Zaragoza. Los resultados indican que aquellas actividades que implican un aprendizaje integral y activo, como la construcción de una historia, repercuten significativamente en el rendimiento académico, además de contribuir a una mayor percepción de utilidad y satisfacción del alumnado. En este sentido, se plantea la idoneidad de generalizar este tipo de herramientas didácticas a otras asignaturas del Grado vinculadas a diferentes áreas de conocimiento.

Palabras clave: narración, historias, storytelling, innovación docente, aprendizaje activo.

Storytelling is a useful tool to promote active learning among university students. This is especially relevant in the field of Labor Relations and Human Resources (LRHR), since the story may introduce specific situations related to human behavior within the workplace. The objective of this research is to analyze the results obtained after applying storytelling tool with 111 students enrolled in a subject of the Degree in LRHR of the University of Zaragoza. The results indicate that those activities that involve an active and integral learning, such as the construction of a story, have a significant impact on the academic outcomes, besides contributing to a greater perception of the students' usefulness and satisfaction. In this sense, it is proposed that storytelling may have similar results in other subjects of LRHR, including those who belong to different areas of knowledge.

Keywords: narration, stories, storytelling, teaching innovation, active learning. 


\section{SUMARIO}

Sumario. 1. Introducción. 2. Método. 2.1. Participantes. 2.2. Procedimiento. 2.3. Instrumentos. 2.4. Análisis de datos. 3. Resultados. 4. Discusión. 5. Bibliografía.

\section{Introducción}

Uno de los aspectos más destacados del Espacio Europeo de Educación Superior (EEES) es, sin duda, el énfasis en la calidad del sistema universitario (European Association for Quality Assurance in Higher Education, 2015: 7). Por ello, entre los criterios para el aseguramiento interno de dicha calidad, se incluye la obligación por parte de las instituciones, de asegurarse que la impartición de los programas de las distintas asignaturas anime al alumnado a involucrarse activamente en el proceso de aprendizaje.

En este sentido, tal y como apunta el Informe elaborado por el Consejo de Coordinación Universitaria (2006: 28), la renovación de las metodologías educativas en la Universidad es clave. Si queremos formar a estudiantes más activos, que puedan comprender y enjuiciar con más profundidad la materia que estudian, debemos utilizar diferentes fórmulas que fomenten su aprendizaje (Prince, 2004: 225), es decir, que mejoren su capacidad para aplicar los conocimientos teóricos a la práctica (Kober, 2015: 61).

Este tipo de aprendizaje activo es esencialmente importante en el ámbito de las Ciencias Sociales, donde se plantean diferentes modelos sobre el comportamiento humano en contextos muy variados y cambiantes que requieren que el alumnado se involucre más en responder y hacer preguntas, en pensar y resolver problemas, o en explicar y reflexionar sobre lo que ha aprendido (Sánchez, 2008: 8). Para la promoción de este tipo de aprendizaje activo, contamos con diversas herramientas, entre las que destaca el storytelling o narración (Flanagan, 2015: 148). La narración, o el también llamado arte de contar historias, es una práctica basada en el paradigma constructivista (Guba y Lincoln, 1990: 80) que asume que el conocimiento es el producto o resultado de la actividad cognitiva del sujeto que aprende a partir de aquellos fenómenos que se quieren conocer.

El storytelling tiene diversos usos. El primero de ellos es servir como medio de investigación social. En este sentido, es muy conocido el estudio de Boje 
(1991: 122), llevado a cabo en una empresa de suministros de oficina, y que puso de manifiesto que los relatos que tenían lugar en ella podían ser escuchados, regulados y controlados. En segundo lugar, tal y como apuntan Leonard y Cook (2010: 101), la utilización de historias o casos fomenta la capacidad de diagnóstico y toma de decisiones en el ámbito social y organizacional, donde las relaciones humanas juegan un papel fundamental. Finalmente, el storytelling puede ser utilizado como herramienta de intervención profesional. Así, algunos autores han apostado por la utilidad de las narraciones para la gestión empresarial (e.g., fomentar iniciativas o comunicar de ideas complejas, compartir información y conocimientos, conseguir que la gente trabaje en equipo, fomentar la confianza, transmitir valores, etc.) o la resolución de problemas organizacionales (Denning, 2005: 12; 2006: 45; Mateo, 2006). Dentro del marco de aprendizaje cooperativo en la educación superior, el storytelling se basa en que los alumnos contextualicen conceptos teóricos de la asignatura a través de la creación de una historia, lo que ofrece una gran oportunidad para promover de forma más activa la construcción colectiva de conocimientos (Kunselman y Johnson, 2004: 92; Piqué y Forés, 2012: 89).

Llegados a este punto cabe plantearse los siguientes interrogantes: ¿Cómo podemos introducir el aprendizaje a través de historias en el ámbito de la educación superior? ¿Realmente contribuye el storytelling a fomentar el aprendizaje activo de los estudiantes universitarios? En general, la evidencia apunta a que usar historias ficticias en el ámbito docente es una estrategia adecuada de enseńanza participativa en las diversas áreas de conocimiento. Así, varios estudios exploratorios han confirmado que la escritura de historias o casos repercute positivamente en las calificaciones finales de la asignatura en la que se han utilizado, en las notas globales de ese curso académico y en la satisfacción global de los estudiantes (Escartín et al., 2015: 49; Jordal y Heggen, 2015: 112; Lenette, Cox y Brough, 2013: 13; Scott, 2007: 31).

$\mathrm{Y}$, ¿qué ocurre en el ámbito de los estudios universitarios sobre Relaciones Laborales y Recursos Humanos (RLRH)? Aunque no hay estudios específicos al respecto, la literatura científica previa en el ámbito organizacional en general y los resultados positivos obtenidos en otras carreras universitarias, apuntan a que el storytelling sería una herramienta relevante en la enseńanza de los estudios universitarios de RLRH, por cuanto el caso creado por los estudiantes se aplicaría a los conceptos y situaciones concretas relacionadas con el comportamiento humano dentro del ámbito laboral.

El objetivo de este trabajo es presentar los resultados obtenidos tras la introducción de la metodología del storytelling o creación de historias en una asignatura de primer curso del Grado en RLRH de la Universidad de Zaragoza. En concreto, se analiza la percepción de utilidad de este tipo de ejercicio por parte del alumnado y su relación con el rendimiento académico. 


\section{Método}

\subsection{Participantes}

La muestra estuvo formada por 111 estudiantes (71,2\% mujeres; $M=21,3$ años, $D T=6,08)$ matriculados en la asignatura de primer curso "Psicología del Trabajo y de las Organizaciones» de la Universidad de Zaragoza durante el primer semestre del curso académico 2016-2017. Únicamente se han tenido en cuenta para el análisis, los datos de aquellos estudiantes que realizaron el ejercicio de storytelling, cumplimentaron todos los cuestionarios relativos a la utilidad de las actividades desarrolladas y se presentaron a las pruebas de evaluación finales en febrero de 2017 (49,77\% del total de alumnos matriculados).

\subsection{Procedimiento}

La construcción de una historia fue introducida como eje central de las actividades prácticas de la asignatura conocidas como "actividades tipo 6" (trabajo práctico tutelado). Los estudiantes trabajaron en pequeños grupos de 3 a 5 personas en la construcción del caso a lo largo del semestre siguiendo las cuatro fases que se describen a continuación.

La primera fase es en la que se presenta el modelo de ejercicio. Para ello, se entregó a los estudiantes un modelo de historia elaborado por los docentes que sirviese como ejemplo o modelo para la elaboración de la suya propia.

A continuación se llevó a cabo la fase dos, creación de la historia. A medida que se iba explicando el programa teórico de la asignatura, cada grupo de trabajo fue construyendo su historia. Los estudiantes tenían que decidir qué conceptos iban a introducir en la misma, pero sin utilizarlos de manera explícita en el texto concreto que tenían que escribir. Así, los estudiantes debían construir una historia real o ficticia, que incluyese y utilizase los contenidos vistos durante las clases magistrales. Cada grupo de trabajo acudió a una tutoría mensual con el profesor responsable para resolver dudas o incidencias puntuales en la construcción de su historia.

La tercera fase fue el análisis de la historia. Además de construir una historia, cada grupo elaboró un informe explicativo, en el cual debían nombrar, describir y justificar adecuadamente qué conceptos habían introducido.

La cuarta y última fase consistió en la exposición de la historia. Al finalizar la elaboración de la historia, cada grupo de trabajo la expuso oralmente al resto de estudiantes. El formato de presentación fue libre (e.g., presentación mediante diapositivas, realización de role-play, etc.) Tras la exposición, los demás estudiantes debían averiguar qué conceptos habían sido introducidos en la historia que acababan de escuchar y justificar su respuesta. 


\subsection{Instrumentos}

Tras la entrega y exposición oral de las historias, para poder analizar la percepción de utilidad de este ejercicio, se administró a cada estudiante un cuestionario con formato de respuesta tipo Likert de 1 (totalmente en desacuerdo) a 5 (totalmente de acuerdo). Este cuestionario, en forma de rúbrica, está basado en el trabajo de Price, Strodtman, Brough, Lonn y Luo (2015: 69) e incluye la evaluación de siete dimensiones: (1) nuevos conocimientos; (2) aprendizaje más profundo; (3) pensamiento crítico; (4) aplicación a la práctica; (5) aplicación a la evaluación; (6) consideración como un buen ejercicio; y, (7) mejor comprensión conceptual. Además, se incluyó un apartado para poder recopilar de forma cualitativa la experiencia de los estudiantes respecto el ejercicio realizado.

Este mismo cuestionario se administró tras la realización de dos ejercicios, llevados a cabo dentro de las actividades prácticas tipo 2 de la asignatura, para poder comparar con la percepción de utilidad del ejercicio de storytelling (ES). Ambas actividades fueron semejantes en forma (actividad práctica en pequeño grupo de 3 a 5 estudiantes) a la actividad de construcción de una historia. El primer ejercicio (E1) consistió en la realización de un análisis de un puesto de trabajo y fue llevado a cabo en las semanas 4 y 5 del semestre. En el segundo ejercicio (E2), los estudiantes, tras la construcción y administración de un cuestionario de satisfacción laboral a 10 trabajadores, elaboraron un pequeño informe con el análisis de los principales resultados que fue entregado en las semanas 7 y 8 del semestre.

En relación al rendimiento académico, éste se operativizó en las calificaciones obtenidas por cada estudiante en las siguientes actividades de la asignatura: (1) en la construcción de la historia (ES); (2) en otros dos ejercicios prácticos como forma de comparación (E1 y E2); (3) en la nota del examen teórico (prueba objetiva con 30 preguntas); (4) en la nota del examen práctico (prueba escrita con cinco preguntas cortas). Las puntuaciones fueron transformadas en una escala de 0 a 10 para facilitar la comparación entre las diferentes actividades.

\subsection{Análisis de datos}

Tras comprobar la ausencia de ajuste a la normalidad mediante la prueba Kolmogorov-Smirnov, se emplearon estadísticos descriptivos (media, desviación típica), diferencias de medias mediante la prueba $Z$ de Wilcoxon para muestras relacionadas y correlaciones de Spearman. Las distribución de las respuestas en los ejercicios E1, E2 y ES se analizó utilizando el diagrama de cajas y bigotes, que facilita la inspección visual de este tipo de información (Weissgerber, Milic, Winham y Garovic, 2015: 4). Finalmente, se llevaron a cabo análisis de regresión jerárquica, adoptando como medida de ajuste el porcentaje de varianza explicado. 
Para el análisis de los datos se ha utilizado el programa estadístico IBM SPSS Statistics 22.

\section{Resultados}

En la tabla 1 se muestran las medias obtenidas en la evaluación de percepción de utilidad de las tres actividades realizadas en la asignatura «Psicología del Trabajo y de las Organizaciones».

Tabla 1

Percepción de utilidad de los ejercicios realizados en la asignatura «Psicología del Trabajo y de las Organizaciones»

\begin{tabular}{l|cc|cc|cc}
\hline \multirow{2}{*}{\multicolumn{1}{c|}{ Ítems }} & \multicolumn{2}{|c|}{$\mathrm{E} 1$} & \multicolumn{2}{c|}{$\mathrm{E} 2$} & \multicolumn{2}{c}{$\mathrm{ES}$} \\
\cline { 2 - 7 } & $M$ & $D T$ & $M$ & $D T$ & $M$ & $D T$ \\
\hline 1. Ha promovido nuevos conocimientos & 4,03 & 0,59 & 4,01 & 0,65 & 4,29 & 0,59 \\
\hline $\begin{array}{l}\text { 2. Ha favorecido un aprendizaje más pro- } \\
\text { fundo }\end{array}$ & 3,95 & 0,75 & 3,83 & 0,62 & 4,40 & 0,62 \\
\hline $\begin{array}{l}\text { 3. Me ha ayudado a pensar de forma más } \\
\text { crítica }\end{array}$ & 3,76 & 0,80 & 3,80 & 0,80 & 3,90 & 0,77 \\
\hline $\begin{array}{l}\text { 4. Me ayudará a aplicar el contenido traba- } \\
\text { jado a la práctica. }\end{array}$ & 4,32 & 0,62 & 4,32 & 0,71 & 4,53 & 0,55 \\
\hline $\begin{array}{l}\text { 5. Me ayudará a aplicar el contenido traba- } \\
\text { jado a la evaluación. }\end{array}$ & 4,20 & 0,70 & 4,11 & 0,67 & 4,56 & 0,53 \\
\hline $\begin{array}{l}\text { 6. Ha sido un buen ejercicio en la asigna- } \\
\text { tura }\end{array}$ & 4,23 & 0,64 & 4,23 & 0,61 & 4,54 & 0,57 \\
\hline $\begin{array}{l}\text { 7. Me ha ayudado a comprender mejor los } \\
\text { conceptos }\end{array}$ & 4,30 & 0,60 & 4,02 & 0,73 & 4,66 & 0,51 \\
\hline
\end{tabular}

En el diagrama de cajas (figura 1), se muestra la mediana de la valoración total en cada uno de los tres ejercicios (E1, E2 y ES), que se comparan entre sí. La caja del diagrama representa el $50 \%$ de los datos, y los bigotes superior e inferior el $50 \%$ restante. Como se puede observar, las valoraciones de los estudiantes en el ejercicio de storytelling se encuentran agrupadas en puntuaciones elevadas (mediana $=4,43)$. 
Figura 1

Valoración de utilidad de los ejercicios en la asignatura «Psicología del Trabajo y de las Organizaciones»

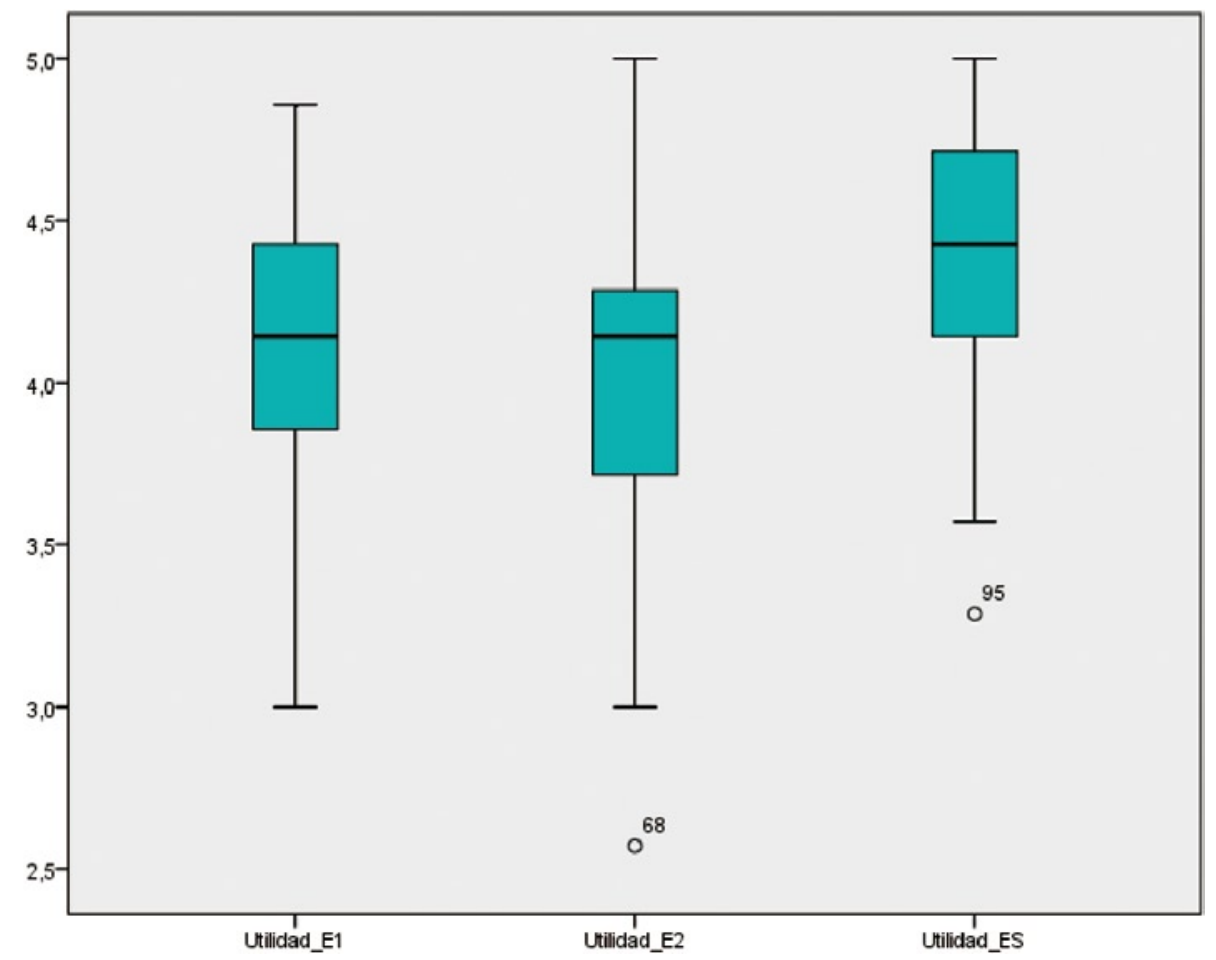

Al comparar los tres ejercicios, encontramos que las diferencias de medias entre el E1 $(M=4,11 ; D T=0,40)$, el E2 $(M=4,04 ; D T=0,43)$ y el ES $(M=4,41 ; D T=0,38)$ fueron estadísticamente significativas ( $Z$ de Wilcoxon $=-6,036, p<, 001 ; Z=-7,129, p<, 001$, respectivamente). El ejercicio de storytelling obtuvo, por tanto, una valoración media global más alta por parte de los estudiantes que las otras dos actividades prácticas. Si analizamos un poco más detalladamente las puntuaciones en cada ítem del cuestionario en cada uno de los tres ejercicios (tabla 1), observamos que los alumnos destacan especialmente que el ES ha favorecido un aprendizaje más profundo (ítem 2) y que les ha ayudado a comprender mejor los conceptos (ítem 7).

Desde un punto de vista cualitativo, a modo de ejemplo, en la tabla 2, se recogen algunos de los comentarios realizados por los estudiantes en relación a la creación de historias. En líneas generales, los alumnos destacan que la creación y narración de una historia vinculada a la asignatura fomenta el aprendizaje ac- 
tivo, así como contribuye al desarrollo de competencias transversales (e.g., trabajo en equipo, expresión oral) y específicas (e.g., conocer, comprender y asimilar mejor los aspectos teóricos fundamentales; aplicar los conocimientos a la práctica, etc.)

\section{Tabla 2}

Opiniones del alumnado de la asignatura

«Psicología del Trabajo y de las Organizaciones»

sobre la utilidad de la creación de historias

\begin{tabular}{l} 
Ejemplo comentario \\
\hline — «Es un modelo educativo diferente, pero que su uso ayuda a desarrollar un aprendi- \\
zaje más activo y a la vez es un proyecto muy participativo en el cual compartes co- \\
nocimientos. Por lo que considero que se tendría que aplicar a más asignaturas». \\
— «Es un trabajo interesante que te ayuda a estudiar de una manera más dinámica y di- \\
vertida junto a los compañeros». \\
— «Es una actividad divertida, he aprendido muchos conceptos y también me ha ser- \\
vido para poder interpretarlos mejor». \\
— «Excelente manera de poner en orden ideas y conceptos». \\
— «La actividad me ha ofrecido la oportunidad de tener la asignatura al día y pensar so- \\
bre los conceptos teóricos para aplicarlos al mundo real. Ha sido una muy buena ex- \\
periencia el trabajo en grupo, para relacionarlos con otras personas y aprender dife- \\
rentes versiones para hacer las cosas y aprender a respetar otras opiniones». \\
— «Me ha parecido un buen trabajo que ayuda a desarrollar todo lo que sabes y a apli- \\
carlo a la vida diaria. De todas las prácticas grupales, esta es la que más ayuda al estu- \\
dio». \\
—Me ha parecido un buen trabajo, tanto como para mejorar el trabajo en equipo \\
como para profundizar y comprender los temas y conceptos de la asignatura». \\
«Me parece un trabajo muy útil y divertido, de este modo aprendes conceptos y sirve \\
de cara a la evaluación». \\
«Pensaba que la actividad iba a ser más difícil pero ha sido una actividad interesante \\
y divertida. Y para representar lo mismo, se puede hacer fácilmente y es bueno para \\
aprender conceptos del temario y compartir opiniones con los compañeros». \\
«Un ejercicio muy dinámico, considero que motiva al alumno no solo a repasar el te- \\
mario sino ponerlo en práctica».
\end{tabular}

Con respecto al rendimiento académico, tal y como aparece en la tabla 3, se encontraron correlaciones positivas entre la calificación obtenida en el ES y las calificaciones obtenidas en el examen teórico $(r=, 36, p<, 001)$ y el examen 
práctico $(r=, 35, p<, 001)$. En cambio, en el caso de los otros dos ejercicios (E1 y E2), únicamente el E1 correlacionó positivamente, con la calificación obtenida en el examen teórico $(r=, 19, p<, 05)$.

Tabla 3

Correlaciones entre los ejercicios realizados en la asignatura «Psicología del Trabajo y de las Organizaciones» y las calificaciones obtenidas en el examen teórico y el examen práctico

\begin{tabular}{|c|c|c|c|c|}
\hline Variables & 1 & 2 & 3 & 4 \\
\hline \multicolumn{5}{|l|}{ 1. E1 } \\
\hline 2. E2 &, $39^{* * *}$ & & & \\
\hline 3. ES &, $34^{* * *}$ &, $30^{* *}$ & & \\
\hline 4. Examen teórico &, $19^{*}$ &, 09 &, $36^{* * *}$ & \\
\hline 5. Examen práctico &, 15 &,- 06 &, $35^{* * *}$ &, $67^{* * *}$ \\
\hline
\end{tabular}

Nota. E1 = ejercicio práctico n. ${ }^{\circ} 1 ; \quad$ E2 $=$ Ejercicio práctico $2 ; \quad$ ES $=$ ejercicio de construcción de la historia.

${ }^{*} p<, 05 ; \quad * * p<, 01 ; \quad * * * p<, 001$.

Finalmente, con el objetivo de conocer si la actividad de storytelling contribuía a explicar las calificaciones finales obtenidas en los exámenes finales, se llevaron a cabo dos análisis de regresión jerárquica. Más concretamente, se elaboraron varios modelos para cada tipo de prueba de evaluación (teórica y práctica), introduciendo como predictores las calificaciones obtenidas en los tres ejercicios grupales propuestos (E1, E2 y ES) siguiendo el orden en el que fueron llevados a cabo durante el semestre. Los resultados obtenidos en cada modelo aparecen recogidos en la tabla 4. Así, en ambas pruebas de evaluación, el único predictor significativo resultó ser la calificación obtenida en el ejercicio de storytelling ( $\beta=, 26$ y $\beta=, 32$, respectivamente), explicando un 7,6\% y $9,7 \%$ de la varianza en el rendimiento académico teórico y práctico. Ninguno de los otros dos ejercicios realizados en la asignatura resultó ser un predictor significativo del rendimiento académico de los estudiantes. 
Tabla 4

Modelos de predicción del rendimiento académico en la asignatura «Psicología del Trabajo y las Organizaciones» realizados con regresión jerárquica

\begin{tabular}{|c|c|c|c|c|c|}
\hline Criterio & Modelo & Predictores & $R^{2}$ & $R^{2}$ corregida & $\beta$ \\
\hline \multirow{3}{*}{$\begin{array}{c}\text { Examen teórico } \\
\text { (calificación obtenida) }\end{array}$} & 1 & Calificación E1 &, 05 & 04 & $22^{*}$ \\
\hline & 2 & $\begin{array}{l}\text { Calificación E1 } \\
\text { Calificación E2 }\end{array}$ & 05 & 03 & $\begin{array}{r}, 23^{*} \\
-, 02\end{array}$ \\
\hline & 3 & $\begin{array}{l}\text { Calificación E1 } \\
\text { Calificación E2 } \\
\text { Calificación ES }\end{array}$ &, 10 & ,08 & $\begin{array}{r}, 18 \\
-, 10 \\
, 26^{*}\end{array}$ \\
\hline \multirow{3}{*}{$\begin{array}{l}\text { Examen práctico } \\
\text { (calificación obtenida) }\end{array}$} & 1 & Calificación E1 & ,04 & ,03 &, $19^{*}$ \\
\hline & 2 & $\begin{array}{l}\text { Calificación E1 } \\
\text { Calificación E2 }\end{array}$ &, 04 & ,02 & $\begin{array}{r}, 23^{*} \\
-, 09\end{array}$ \\
\hline & 3 & $\begin{array}{l}\text { Calificación E1 } \\
\text { Calificación E2 } \\
\text { Calificación ES }\end{array}$ & , 12 &, 10 & $\begin{array}{r}, 17 \\
-, 20 \\
, 32^{* *}\end{array}$ \\
\hline
\end{tabular}

Nota. ${ }^{*} p<, 05 ; \quad * * p<, 01$.

\section{Discusión}

El presente trabajo tenía como objetivo analizar los resultados obtenidos tras introducir el storytelling, en términos de percepción de utilidad y rendimiento académico de los estudiantes, en la asignatura "Psicología del Trabajo y de las Organizaciones» del Grado en RLRH de la Universidad de Zaragoza.

Para llevar a cabo este análisis se ha comparado la valoración realizada por los estudiantes de la construcción de una historia frente a otros dos ejercicios, y se ha evaluado el impacto de estas actividades en el rendimiento académico. Los resultados obtenidos confirman que la metodología basada en el storytelling no solo es percibida por los alumnos del Grado en RLRH como una herramienta útil para su proceso de aprendizaje, sino que además contribuye a explicar sus calificaciones finales en la asignatura, en la línea de lo que se ha encontrado estudios previos (Escartín et al., 2015: 49; Flanagan, 2015: 164; Scott, 2007: 31).

La valoración media del ejercicio de creación de historias en términos de utilidad fue alta en comparación con las otras actividades grupales llevadas a cabo en la asignatura. Así, los alumnos destacan que se trata de un buen ejercicio, que 
permite mejorar la comprensión de los conceptos vistos durante las clases magistrales y aplicar el contenido a la evaluación, lo que redunda en un aprendizaje más profundo. En este sentido, Price et al. (2015: 69) encontraron en su estudio cómo el storytelling en las aulas universitarias incrementaba su atención, promovía nuevos conocimientos y aumentaba la satisfacción global de los alumnos con esa asignatura. Además, la creación y narración de historias puede contribuir a mejorar el desarrollo de algunas competencias transversales que son requeridas en la educación superior, como son el trabajo en equipo, la creatividad o la motivación, a la vez que los alumnos disfrutan por su capacidad de entretener (Moorman, 2015). En los comentarios cualitativos de los estudiantes que han participado en este estudio ha quedado reflejado cómo este tipo de herramienta educativa no sólo ayuda a la comprensión de conceptos, sino que permite hacerlo de una manera más amena y divertida.

La actividad de crear una historia en el contexto de la asignatura «Psicología del Trabajo y las Organizaciones» del Grado en RLRH tuvo además un impacto significativo en el rendimiento académico de los estudiantes. Así, el ejercicio de storytelling correlacionó positivamente con la calificación del examen teórico y el examen práctico, mientras que ninguna de las dos otras actividades lo hicieron de manera significativa (a excepción del E1 con el examen teórico). Estos resultados, junto a los obtenidos en los análisis de regresión realizados, nos permiten inferir que el rendimiento académico puede mejorar a medida que el estudiante se convierte en un participante activo de su propio aprendizaje. En este sentido, se plantea la idoneidad de generalizar este tipo de actividades a otras asignaturas del Grado en RLRH vinculadas a diferentes áreas de conocimiento (e. g., Sociología, Derecho del Trabajo, Organización de Empresas, etc.).

No obstante, el presente trabajo no está exento de varias limitaciones. La primera de ellas tiene que ver con la ausencia de un grupo control que permitiese comprobar el efecto real de la creación y narración de la historia en el rendimiento académico de los estudiantes. Asimismo, la propia naturaleza de la muestra de participantes (estudiantes de primer curso del Grado en RLRH) impide la generalización de los resultados obtenidos a alumnos de otras titulaciones universitarias. Por otro lado, el tamaño muestral se ha visto reducido significativamente dado que solo se analizaron los datos de aquellos estudiantes que llevaron a cabo los tres ejercicios, cumplimentaron los cuestionarios de percepción de utilidad después de cada uno de ellos y se presentaron a las pruebas de evaluación finales. En cuarto lugar, la metodología del storytelling fue introducida en un tipo de actividad (trabajo práctico tutelado o tipo 6) distinto a las actividades en las que se llevaron a cabo los dos ejercicios de comparación (actividades prácticas tipo 2). Mientras que el trabajo práctico tutelado se desarrolla a lo largo de todo el semestre y se acompaña de una tutorización mensual por parte del profesorado, las actividades prácticas tipo 2 se llevan a cabo en dos momentos concretos del curso y únicamente reciben soporte de los docentes en caso de 
que los estudiantes lo soliciten de manera expresa. Teniendo en cuenta esto, y según lo obtenido en los análisis de regresión, no podemos descartar la existencia de un posible efecto acumulativo sobre el aprendizaje de los estudiantes, lo cual puede haber influido en los resultados encontrados.

En base a todo lo anterior y de cara a la investigación futura, sería interesante llevar a cabo un estudio longitudinal, con grupo control, con el que comprobar si efectivamente la metodología del storytelling contribuye al desarrollo y el mantenimiento a largo plazo de competencias transversales y específicas de la titulación en la que se introduce. Además, sería interesante incluir otro tipo de predictores, como por ejemplo los procesos que tienen lugar en los equipos de trabajo, en la medida en que pueden afectar significativamente al rendimiento de los estudiantes. Asimismo, se considera necesario llevar a cabo estudios interdisciplinares, en diferentes áreas de conocimiento y distintas titulaciones, para comprobar realmente la utilidad del storytelling en la promoción del aprendizaje activo en la educación superior.

\section{Bibliografía}

BoJe, David (1991): "The storytelling organization: a study of story performance in an office-supply firm", Administrative Science Quarterly, 36, 106-126.

Consejo de Coordinación Universitaria (2006): Propuestas para la renovación de las metodologías educativas en la universidad, Madrid, Ministerio de Educación y Ciencia.

Denning, Stephen (2005): La estrategia de la ardilla: El poder de las fábulas como motor del cambio empresarial, Barcelona, Empresa Activa.

Denning, Stephen (2006): «Effective storytelling: strategic business narrative techniques», Strategy \& Leadership, 34, 42-48.

Escartín, Jordi, Saldaña, Omar, Martín-Peña, Javier, Varela-Rey, Ana, Jiménez, Yirsa, Vidal, Tomeu y Rodríguez-Carballeira, Álvaro (2015): "The impact of writing case studies: benefits for students' success and well-being», Procedia-Social and Behavioral Sciences, 196, 47-51.

European Association for Quality Assurance in Higher Education (2015): Criterios y directrices para el Aseguramiento de la Calidad en el Espacio Europeo de Educación Superior (ESG), Agencia Nacional de Evaluación de la Calidad y Acreditación, ANECA.

FLANAGAN, Sarah (2015): «How does storytelling within higher education contribute to the learning experience of early years students?», Journal of Practice Teaching \& Learning, 13, 146-168.

Guba, Egon y Lincoln, Yvonna (1990): Fourth generation evaluation (2a. ed.), London, Sage.

Jordal, Kristin y Heggen, Kristin (2015): «When life experience matters: A narrative exploration of students' learning in nursing education», Nordic Psychology, 67, 104-116.

KobER, Nancy (2015): Reaching students: What research says about effective instruction in undergraduate Science and Engineering, Washington, The National Research Council's Division on Behavioral and Social Sciences and Education. 
Kunselman, Julie y Johnson, Kathrine (2004): "Using the case method to facilitate learning», Collage Teaching, 52, 87-92.

Lenette, Caroline, Cox, Leonie y Brough, Mark (2013): «Digital storytelling as a social work tool: learning from ethnographic research with women from refugee backgrounds", British Journal of Social Work, 1-18.

LeOnARD, Edwin y Cook, Roy (2010): "Teaching with Cases», Journal of Teaching in Travel \& Tourism, 10, 95-101.

Mateo, Juan (2006): Cuentos que mi jefe nunca me contó, Madrid, LID.

Moorman, Diann (2015): «This is not a fable: using storytelling in a college classroom to enhance student learning», SoTL Commons Conference, Georgi, Georgia Southern University.

Piqué, Begoña y Forés, Anna (2012): Propuestas metodológicas para la educación superior, Barcelona, Universitat de Barcelona.

Price, Deborah, Strodtman, Linda, Brough, Elizabeth, Lonn, Steven y Luo, Airong (2015): «Digital storytelling: an innovative technological approach to nursing education", Nurse Educator, 40, 66-70.

Prince, Michael (2004): «Does active learning work? A review of the research», Journal of Engineering Education, 93, 223-231.

SÁNChEZ, Marita (2008): Como enseñar en las aulas universitarias a través del estudio de casos, Zaragoza, Instituto de Ciencias de la Educación, Universidad de Zaragoza.

Scotт, Noel (2007): «An evaluation of the effects of using case method on student learning outcomes in a tourism strategic planning course», Journal of Teaching in Travel \& Tourism, 7, 21-34.

Weissgerber, Tracey, Milic, Natasa, Winham, Stacey y Garovic, Vesna (2015): «Beyond bar and line graphs: Time for a new data presentation paradigm", PLOS Biology, 1-10. 